\title{
Supporting Secure Provenance Update by Keeping "Provenance" of the Provenance
}

\author{
Amril Syalim ${ }^{1}$, Takashi Nishide ${ }^{2}$, and Kouichi Sakurai ${ }^{2}$ \\ 1 Fakultas Ilmu Komputer, Universitas Indonesia \\ amril.syalim@cs.ui.ac.id \\ 2 Department of Informatics, Kyushu University, Fukuoka, Japan \\ \{nishide, sakurai\}@inf .kyushu-u.ac.jp
}

\begin{abstract}
Provenance of data is a documentation of the origin and processes that produce the data. Many researchers argue that the provenance should be immutable: once a provenance is submitted, it should not be changed or updated. A main reason is that the provenance represents the history of data, and the history should not be altered or changed because it represents the fact in the past. Provenance can be represented by a graph, where each node represents the process executed by a party and an edge represents the relationship between two nodes (i.e. a child node uses the outputs of the parent nodes). A method to ensure that the provenance has not been updated is by using signature chain, where the signatures of the parent nodes are recorded in the children nodes so that any changes to the parent nodes will raise inconsistencies between the parent and the children. However, sticking to the requirement that the provenance should be immutable requires unlimited data storage and also we have problems whenever we need to update the provenance for an accidental error. In this paper, we propose a method that allows updates in the signature chain-based secure provenance, while keeping the signature consistent. The main idea is by keeping the "provenance" of the provenance itself, that is the history of update of the provenance, in the form of the signatures of the previous versions of the nodes. We implement the idea by keeping the signatures of the previous version in a signature tree similar to the Merkle-tree, where the a parent node in tree is the aggregate signature of the children. Using this method, the storage requirement to store signatures is always smaller than the number of updates.
\end{abstract}

Keywords: Provenance, Provenance Security, E-science, Data lineage.

\section{Introduction}

Provenance is a documentation of data history. It records the processes that produce the data and relationship between the processes. A simple example of the provenance is the documentation about how to produce a patient record in a hospital [2]6[12[13. The patient record contains the data about the medical treatment or medical test of the patient which is produced by the physicians and laboratory staffs in the hospital. In this case the processes to produce data are executed by the physicians or the laboratory staffs. A process may have relationships with the other processes, for example: to produce a medical treatment, a 
physician uses the data produced by another physicians (i.e. medical diagnosis) or data used by a laboratory staffs (i.e. a result of blood or urine test of the patient).

Provenance can be represented by a graph [147/15, where the nodes represent the processes and the data while the edges represent relationships between processes. An example of the graph model for provenance is the open provenance model which is proposed as a standard for provenance [16 15]. The open provenance model specification defines some types of the nodes (i.e. actor, process and artifacts), and some types of the relationships between the nodes.

Some of the the important research problems with the provenance are related to security 211011121920. The security problems are caused by malicious update and deletion to the provenance. For example in a hospital, if we allow update to the provenance and the patient record, there can be inconsistencies between provenance and data created by a physician with another physician or laboratory staffs. A physician $A$ may decide a medical treatment based on the diagnosis created by physician $B$, or medical test produced by a laboratory staff $C$. If $B$ or $C$ updates/deletes the provenance of diagnosis or medical test, the treatment by $A$ may be incorrect because it is based on the previous versions of the diagnosis or medical tests produced by $B$ or $C$. If the patient who takes the medical treatment complains about a misconduct by $A, A$ cannot refer to $B$ or $C$ to explain why he/she decides the treatment. In an extreme case, it is possible $B$ or $C$ may maliciously/intentionally try to frame $A$ for a misconduct charge.

\subsection{Problem Definition}

As a history record, many researchers argue that the provenance should be immutable (no change is allowed in the history records) [51815 16 87 17]. However, without being able to update a submitted provenance, the provenance storage is always growing (indefinitely). In this paper, we try to address the problem on how to allow updates/deletes the provenance to save spaces on the storages, while keeping the provenance consistent. The methods are useful in the case the actors who produce the provenance honestly update the provenance for an accidental/unintentional errors and in the case the storage is limited. The main security requirement is: no actor is allowed to exploit the update/deletion features to do a malicious behaviors or avoid responsibilities for the data produced in the past.

\subsection{Contributions}

The idea of our solution is by keeping the "provenance" of the provenance, where using this method, we allow updates/deletions to the provenance but we keep the history of the previous versions of the provenance in the forms of the signatures of the previous versions. So that, even if an actor has updated the provenance and the data, the actor cannot reject the previous versions of the provenance and data. To save the spaces, we store the signatures in a tree similar to the Merkle-tree and combines the signatures using the aggregate signatures techniques. Using this method, for any number of updates the grow of signatures 
storage for the previous versions of the provenance and data is much smaller than the normal updates in the provenance (where we should keep all signatures and data of the previous versions).

\section{Related Work}

\subsection{Hash/Signature Chain, Stamping and Countering}

The integrity scheme to timestamp digital documents using hash/signature chain was first proposed by Haber el al. 9]. It uses a Trusted Timestamping Service (TTS) that issues signed timestamps and also links two timestamps requested consecutively. The TSS links two timestamps by storing the hash value of the first timestamp in the second timestamp. Any changes to the first timestamp can be detected by checking the hash value in the second timestamp. This method is applied by Hasan et al. 12 to a chain model of the provenance where the provenance is modeled as a chain. Aldeco-Perez et al. 1] and Syalim et al. 20 19] extend the hash/signature chain to the provenance graph model.

To detect the problem of the deletion to a provenance node, Syalim et al. proposed countering provenance [20]. The basic idea is a Trusted Counter Server (TCS) assigns a unique consecutive counter number for each node in the provenance graph, so that any deletion to the nodes can be detected from the missing counter number in the nodes. To detect deletion of the newest node, the TCS should store the latest counter number in a trusted storage.

\subsection{Aggregate Signatures}

Aggregate signatures is a technique to combine signatures on many different messages into a short signature. Some aggregate signatures have restriction that they can only be verified if there is no duplicate messages or public keys. However, it is possible to develop a scheme that does not have any restriction [3].

Aggregate signatures can be implemented using bilinear maps [4], that is with the requirement of the existence of a mapping between groups for example the map $e: G_{1} \times G_{2} \rightarrow G_{T}$ where $\left|G_{1}\right|=\left|G_{2}\right|=\left|G_{T}\right|$ with bilinear (for all $u \in G_{1}, v \in G_{2}$ and $\left.a, b \in \mathbb{Z}, e\left(u^{a}, v^{b}\right)=e(u, v)^{a b}\right)$ and non-degenerate $\left(e\left(g_{1}, g_{2}\right) \neq 1\right)$ properties. A particular aggregate signature scheme proposed by Boneh et al [4] is as follows:

Key Generation the user picks random secret key $x \stackrel{R}{\leftarrow} \mathbb{Z}_{p}$ and computes the public key $v \leftarrow g_{2}^{x}$

Signing to sign a message $M$, compute $h \leftarrow H(M)$, where $h \in G_{1}$, and the signature $\sigma \leftarrow h^{x}$.

Aggregation for a set of signatures $\left\{\sigma_{1}, \sigma_{2}, \ldots, \sigma_{k}\right\}$, compute the aggregate signature $\sigma \leftarrow \prod_{i=1}^{k} \sigma_{i}$.

Aggregate Verification for all users $u_{i} \in U$ with public keys $v_{i} \in G_{1}$ and the original messages $M_{i}$, computes $h_{i} \leftarrow H\left(M_{i}\right)$ and accept if $e\left(\sigma, g_{2}\right)=$ $\prod_{i=1}^{k} e\left(h_{i}, v_{i}\right)$ holds. 


\section{Preliminaries}

\subsection{Definition}

Definition 1 (Provenance definition). Provenance related to the data set $D=\left\{d_{0}, d_{1}, \ldots, d_{n-1}\right\}$ stored in a database $D B$ for $n>0$ is a set of provenance nodes $P=\left\{p_{0}, p_{1}, \ldots, p_{n-1}\right\}$ and a binary relation $E$ recorded in a Provenance Store PS. A provenance node $p_{i}$ consists of an identification PID, a process description $A$, a process executor $C I D$, the list of references to a set of inputs $\left\{\operatorname{ref}\left(I_{i}\right)\right\}$, for $I \subseteq D$ and a reference to an output ref $\left(d_{i}\right)$ for $d_{i} \in D$. E is a binary relation in PIDSET where PIDSET is a set of PID of all provenance nodes. E represents the provenance edges such that for $(x, y) \in P I D S E T \times$ $P I D S E T$ and for $x \neq y$ the process documented in provenance node with PID $y$ takes the output of process documented in node with PID $x$ as its input.

In this definition, we store four kind of information about the process execution in the provenance: the description of the process, the information about the process executor, the list of the inputs and an output.

\subsection{Participants in the Provenance System}

The provenance system consists of the following participants:

- A data storage $D B$, where the data is stored

- A provenance storage $P S$, where the provenance of data is stored for a long term storage

- Process executors $C$ with identification $C I D$ is the actors who execute the process, produce the data, submit data to $D B$, and submit the provenance to $P S$

- An auditor $A D T$, is the actor who checks the integrity of the provenance and data

\subsection{The Basic Provenance Recording Method}

A simple model of the provenance recording system is as follows. The process executor $C_{i}$ queries the inputs $I \subseteq D$ from $D B$ and a set of $\left\{P I D_{i n}\right\}$, that is the provenance ID of $I . C_{i}$ executes the process that produces the data output $d_{i}$, creates the provenance node $p_{i}$ and a set of edges, stores $d_{i}$ to $D B$ and submits provenance node $p_{i}$ and edges to $P S$. The provenance edges are a set of mapping from the $P I D$ of provenance $p_{i}$ to the parents that produce $I$, so we write the edges as $\left\{P I D, P I D_{i n}\right\}$. We define the simple protocol as follows [Note: we use the index ${ }_{i n}$ to refer to the parents that produce the inputs $I$, for example $P I D_{i n}$ is a $P I D$ of the parent, $C_{i n}$ is a process executor at the parent]:

$$
\begin{aligned}
D B \rightarrow C_{i} & : I \\
P S \rightarrow C_{i} & :\left\{P I D_{i n}\right\} \\
C_{i} \rightarrow D B & : d_{i} \\
C_{i} \rightarrow P S & : \text { node }=p_{i}, \text { edge }=\left\{P I D, P I D_{i n}\right\}
\end{aligned}
$$




\subsection{Aggregate Signature Aggr and TreeAggr}

We define a function Aggr which aggregates the signatures of different messages created by one or many different parties into one signature. A function TreeAggr aggregates the signatures into a set of signatures where the number of element is less or equal to the total number of the original signatures. The method to aggregate the signatures using TreeAggr is explained in Section 5]

\section{Integrity Scheme for Provenance by Keeping "The Provenance" of the Provenance}

\subsection{Provenance Recording: Securing Provenance with Signature-Chain}

For a secure provenance recording, when querying inputs $I$ and the provenance of the inputs $P I D_{i n}$, the process executor also queries the collection of signature of inputs, that is the signnode in all parent nodes. In the scheme, we include a new participant, a Trusted Provenance Mediator (TPM), which is a trusted party who mediates the provenance recording. We assume that this party is trusted and will not cheat for any purposes. The complete provenance recording protocol is as follows:

$$
\begin{aligned}
D B \rightarrow C_{i}: & I \\
P S \rightarrow C_{i}: & \left\{\text { PID }_{\text {in }}, \text { Sign }_{C_{i n}}\left(p_{i n}, d_{i n}\right)\right\} \\
C_{i} \rightarrow D B: & d_{i} \\
C_{i} \rightarrow T P M: & \text { Insert }\left(\text { node }=p_{i}, \text { edge }=\left\{P I D, P I D_{i n}\right\},\right. \\
& \text { signnode }=\operatorname{Sign}_{C_{i}}\left(p_{i}, d_{i}\right), \\
& \text { signedge } \left.=\left\{\operatorname{Sign}_{C_{i n}}\left(p_{i n}, d_{i n}\right)\right\}\right) \\
T P M \rightarrow P S: & \text { node }=p_{i}, \text { edge }=\left\{P I D, P I D_{i n}\right\}, \\
& \text { signnode }=\operatorname{Sign}_{C_{i}}\left(p_{i}, d_{i}\right), \\
& \text { signedge }=\left\{\operatorname{Sign}_{C_{i n}}\left(p_{\text {in }}, d_{i n}\right)\right\}, \\
& \text { signtpm }=\operatorname{Sign}_{T P M}(\text { signnode }, \text { signedge })
\end{aligned}
$$

The main difference of this scheme with the basic provenance recording method in Section 3.3 is we include signature chain to the provenance, by recording the signature of the node (signnode) and signatures of all edges that connect the node to the parents (signedge - represented by the parent signatures). The signatures are signed by the TPM before submitted to the provenance store $P S$ (signtpm).

\subsection{Provenance Update: Allowing Update in the Signature Chain}

Update to a node by the same process executor is allowed, but we should keep the aggregate of the previous versions of the node. For example, $C_{i}$ updates $p_{i}$ 
to $p_{i}^{\prime}$ and $d_{i}$ to $d_{i}^{\prime}$ and no update to the edges, so that no change to signedge. This update will delete the previous version of $p_{i}$, and change it to $p_{i}^{\prime}$. However it records the signatures of the previous versions in the form of an aggregate signature.

$$
\begin{aligned}
C_{i} \rightarrow D B: & \operatorname{Update}\left(d_{i}^{\prime}\right) \\
C_{i} \rightarrow T P M: & \operatorname{Update}\left(\text { node }=p_{i}^{\prime}, \text { edge }=\left\{P I D, P I D_{\text {in }}\right\},\right. \\
& \text { signnode }=\operatorname{Sign}_{C_{i}}\left(p_{i}^{\prime}, d_{i}^{\prime}\right), \\
& \text { signedge } \left.=\left\{\operatorname{Sign}_{C_{i n}}\left(p_{i n}, d_{i n}\right)\right\}\right) \\
T P M \rightarrow P S: & \text { node }=p_{i}^{\prime}, \text { edge }=\left\{P I D, P I D_{\text {in }}\right\}, \\
& \text { signnode } \left.=\operatorname{Aggr}\left\{\operatorname{Sign}_{C_{i}}\left(p_{i}, d_{i}\right)\right\}, \operatorname{Sign}_{C_{i}}\left(p_{i}^{\prime}, d_{i}^{\prime}\right)\right), \\
& \text { signedge }=\left\{\operatorname{Sign}_{C_{i n}}\left(p_{i n}, d_{i n}\right)\right\}, \\
& \text { signtpm }=\operatorname{Sign}_{T P M}(\text { signnode }, \text { signedge })
\end{aligned}
$$

The second update by $C_{i}$ changes $p_{i}^{\prime}$ to $p_{i}^{\prime \prime}$ and $d_{i}^{\prime}$ to $d_{i}^{\prime \prime}$ and no change to signedge.

$$
\begin{aligned}
C_{i} \rightarrow D B: & \operatorname{Update}\left(d_{i}^{\prime \prime}\right) \\
C_{i} \rightarrow T P M: & \operatorname{Update}\left(\text { node }=p_{i}^{\prime \prime}, \text { edge }=\left\{P I D, P I D_{i n}\right\},\right. \\
& \text { signnode }=\operatorname{Sign}_{C_{i}}\left(p_{i}^{\prime \prime}, d_{i}^{\prime \prime}\right), \\
& \text { signedge } \left.=\left\{\operatorname{Sign}_{C_{i n}}\left(p_{i n}, d_{i n}\right)\right\}\right) \\
T P M \rightarrow P S: & \text { node }=p_{i}^{\prime \prime}, \text { edge }=\left\{P I D, P I D_{\text {in }}\right\}, \\
& \text { signnode } \left.\left.=\operatorname{Aggr}_{\operatorname{Sign}}\left(\operatorname{Sign}_{C_{i}}\left(p_{i}, d_{i}\right), \operatorname{Sign}_{C_{i}}\left(p_{i}^{\prime}, d_{i}^{\prime}\right)\right)\right\}, \operatorname{Sign}_{C_{i}}\left(p_{i}^{\prime \prime}, d_{i}^{\prime \prime}\right)\right) \\
& \text { signedge }=\left\{\operatorname{Sign}_{C_{i n}}\left(p_{i n}, d_{i n}\right)\right\} \\
& \text { signtpm }=\operatorname{Sign}_{T P M}(\text { signnode }, \text { signedge })
\end{aligned}
$$

We can accept any number of updates, however we only store the signatures of the previous versions of the nodes whose outputs are used by at least one child node.

\subsection{The Case of Updates of the Parent Nodes}

In Section 4.2, we assume no updates to signedge which means that no change to the input used by the node. However, a child may update the parent to the newest outputs of the parent. In this case, the child should keep the signature of the previous version of the parent using TreeAggr. For example, $C_{i n}$ updates $p_{i n}$ to $p_{i n}^{\prime}$ and $d_{i n}$ to $d_{i n}^{\prime}$. $C_{i n}$ does exactly the same update as described in Section 4.2. After $C_{i n}$ updates the provenance and data, $C_{i}$ also wants to update the parent to the newest version. So, $C_{i}$ update signedge to $\left\{\operatorname{Sign}_{C_{i n}}\left(p_{i n}^{\prime}, d_{i n}^{\prime}\right)\right\}$. The scheme to update the provenance in this case is as follows. 


$$
\begin{aligned}
& D B \rightarrow C_{i}: I \\
& P S \rightarrow C_{i}:\left\{P I D_{i n}, \operatorname{Sign}_{C_{i n}}\left(p_{i n}^{\prime}, d_{i n}^{\prime}\right)\right\} \\
& C_{i} \rightarrow D B: \operatorname{Update}\left(d_{i}^{\prime}\right) \\
& C_{i} \rightarrow T P M: \text { Update }\left(\text { node }=p_{i}^{\prime} \text {, edge }=\left\{P I D, P I D_{i n}\right\},\right. \\
& \text { signnode }=\operatorname{Sign}_{C_{i}}\left(p_{i}^{\prime}, d_{i}^{\prime}\right) \text {, } \\
& \text { signedge } \left.=\left\{\operatorname{Sign}_{C_{i n}}\left(p_{i n}^{\prime}, d_{i n}^{\prime}\right)\right\}\right) \\
& T P M \rightarrow P S: \text { node }=p_{i}^{\prime} \text {, edge }=\left\{P I D, P I D_{i n}\right\}, \\
& \text { signnode } \left.=\operatorname{Aggr}\left\{\operatorname{Sign}_{C_{i}}\left(p_{i}, d_{i}\right)\right\}, \operatorname{Sign}_{C_{i}}\left(p_{i}^{\prime}, d_{i}^{\prime}\right)\right), \\
& \text { signedge }=\left\{\text { TreeAggr }\left\{\operatorname{Sign}_{C_{i n}}\left(p_{i n}, d_{i n}\right), \operatorname{Sign}_{C_{i n}}\left(p_{i n}^{\prime}, d_{i n}^{\prime}\right)\right\}\right\} \text {, } \\
& \text { signtpm }=\operatorname{Sign}_{T P M}(\text { signnode }, \text { signedge })
\end{aligned}
$$

In this case, we aggregate all versions of signedge using TreeAggr.

\subsection{Signature Verification}

To verify consistency between a node and all of its children, the auditor $A D T$ queries the signnode on the parent and all signedge(s) on the children. The $A D T$ combines the signedge(s) to get an aggregate signatures of the parent and compares the result with signnode. A detailed example is described in Section 5 .

\section{$5 \quad$ Aggregating Signatures of Previous Versions of a Provenance Node Using TreeAggr}

In the provenance, we use the aggregate signature to save the spaces needed for storing the signatures of all previous versions of the provenance nodes. Rather than verifying the aggregate signatures using the messages and the public key of the signers, we verify the signatures by comparing the aggregate signatures stored at a node with the aggregate of all signatures stored at all children of the nodes (we cannot verify the signatures by checking the messages - the previous versions of the provenance - because they have been deleted to save the spaces in each update). The motivation to use TreeAggr is because each child may not store all versions of the parent. So, if we use normal aggregate signatures Aggr to save the spaces, and stores the aggregates in each child, we may not be able to combine the signatures to form a full aggregate of all versions of the parent.

Using TreeAggr, at first we represent all versions of the parent in all leave nodes in the signature tree. The child can aggregate the signatures of two consecutive versions (even and odd) in the leave nodes and stores the aggregate in the parent of the leaves. The same method is applied for each level of nodes in the tree to combine the signatures into a smaller number of signatures stored in the nodes at the higher level until we cannot get two consecutive nodes to aggregate.

We give an example of the aggregation as follows. The node $Q$ created by a process executor $C$ has four versions: $Q, Q^{\prime}, Q^{\prime \prime}, Q^{\prime \prime \prime}$, it has 6 children $R, S, T, U, V$, $W . R$ and $S$ use the output of the first, second and third versions of $Q$, while $T$ and $U$ only uses the output of the second version. $V$ and $W$ use the output of 
the third version of $Q$. After sometimes, the children nodes $S, U$ and $W$ update the parent version to the latest version $\left(Q^{\prime \prime \prime}\right)$.

In the above case, in $Q$, we store the aggregate signatures of the previous version of $Q$, that is $\operatorname{Aggr}\left\{\operatorname{Sign}_{C}(Q), \operatorname{Sign}_{C}\left(Q^{\prime}\right), \operatorname{Sign}_{C}\left(Q^{\prime \prime}\right\}\right.$ and the latest signatures $\left(\operatorname{Sign}_{C}\left(Q^{\prime \prime \prime}\right)\right)$. For each child, we store the TreeAggr of all the parent signatures of the child. So, that for each child we store the signatures as follows:

$$
\begin{aligned}
& \text { signedge }_{R}=\text { TreeAggr }\left\{\operatorname{Sign}_{C}(Q), \operatorname{Sign}_{C}\left(Q^{\prime}\right), \operatorname{Sign}_{C}\left(Q^{\prime \prime}\right)\right\}
\end{aligned}
$$

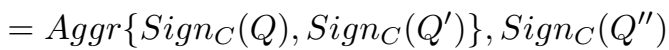

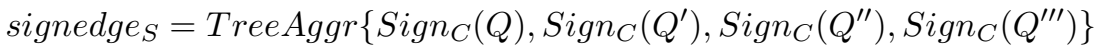

$$
\begin{aligned}
& =\operatorname{Aggr}\left\{\operatorname{Sign}_{C}(Q), \operatorname{Sign}_{C}\left(Q^{\prime}\right)\right\}, \operatorname{Aggr}\left\{\operatorname{Sign}_{C}\left(Q^{\prime \prime}\right), \operatorname{Sign}_{C}\left(Q^{\prime \prime \prime}\right)\right\} \\
& =\operatorname{Aggr}\left\{\operatorname{Sign}_{C}(Q), \operatorname{Sign}_{C}\left(Q^{\prime}\right), \operatorname{Sign}_{C}\left(Q^{\prime \prime}\right), \operatorname{Sign}_{C}\left(Q^{\prime \prime \prime}\right)\right\} \\
& \text { signedge }_{T}=\text { TreeAggr }\left\{\operatorname{Sign}_{C}\left(Q^{\prime}\right)\right\} \\
& =\operatorname{Sign}_{C}\left(Q^{\prime}\right) \\
& \text { signedge }_{U}=\operatorname{TreeAggr}\left\{\operatorname{Sign}_{C}\left(Q^{\prime}\right), \operatorname{Sign}_{C}\left(Q^{\prime \prime \prime}\right)\right\} \\
& =\operatorname{Sign}_{C}\left(Q^{\prime}\right), \operatorname{Sign}_{C}\left(Q^{\prime \prime \prime}\right) \\
& \text { signedge }_{V}=\text { TreeAggr }\left\{\operatorname{Sign}_{C}\left(Q^{\prime \prime}\right)\right\} \\
& =\operatorname{Sign}_{C}\left(Q^{\prime \prime}\right) \\
& \text { signedge }_{W}=\text { TreeAggr }\left\{\operatorname{Sign}_{C}\left(Q^{\prime \prime}\right), \operatorname{Sign}_{C}\left(Q^{\prime \prime \prime}\right)\right\} \\
& =\operatorname{Aggr}\left\{\operatorname{Sign}_{C}\left(Q^{\prime \prime}\right), \operatorname{Sign}_{C}\left(Q^{\prime \prime \prime}\right)\right\}
\end{aligned}
$$

To check the integrity of the child nodes, we aggregate the signatures in some child nodes and compare the aggregate with the aggregate signatures on the parent. The parent $Q$ stores an aggregate of the previous and the latest versions in signnode $=\operatorname{Aggr}\left\{\operatorname{Sign}_{C}(Q), \operatorname{Sign}_{C}\left(Q^{\prime}\right), \operatorname{Sign}_{C}\left(Q^{\prime \prime}\right\}, \operatorname{Sign}_{C}\left(Q^{\prime \prime \prime}\right)\right.$. Because the aggregation can be performed incrementally we may aggregate the signatures into

$$
\begin{aligned}
\text { signnode } & =\operatorname{Aggr}\left\{\operatorname{Aggr}\left\{\operatorname{Sign}_{C}(Q), \operatorname{Sign}_{C}\left(Q^{\prime}\right), \operatorname{Sign}_{C}\left(Q^{\prime \prime}\right)\right\}, \operatorname{Sign}_{C}\left(Q^{\prime \prime \prime}\right)\right\} \\
& =\operatorname{Aggr}\left\{\operatorname{Sign}_{C}(Q), \operatorname{Sign}_{C}\left(Q^{\prime}\right), \operatorname{Sign}_{C}\left(Q^{\prime \prime}\right), \operatorname{Sign}_{C}\left(Q^{\prime \prime \prime}\right)\right\}
\end{aligned}
$$

We can compare the aggregate signatures on children with signnode stored at the parent by finding correct combination of the tree-aggregate on the children to form the same aggregate signature as stored in signode. Some examples of the correct combinations are shown as follows (note: we get $\operatorname{Sign}_{C}\left(Q^{\prime \prime \prime}\right)$ from signedge $\left._{U}\right)$ :

$$
\begin{aligned}
\text { signnode } & =\operatorname{Aggr}_{\left\{\text {signedge }_{R}, \operatorname{Sign}_{C}\left(Q^{\prime \prime \prime}\right)\right\}} \\
& =\text { signedge }_{S}
\end{aligned}
$$

\section{Security Analysis}

To show that a child is a node that uses an output of a version of the parent, the auditor $A D T$ should query all signedge of all children of the node, find a combination of signatures that can reproduce signnode and compare with 
signnode on the parent. Using the scheme explained in the previous versions, it is always possible to find a combination of signatures in the children of a node which produce exactly the same signature as signnode.

Theorem 1. The Auditor ADT can always find a combination of the signatures singedge on the children which produce signnode in the parent.

Proof. The parent node stores the signatures of the latest version and the signatures of the previous versions of the node, except for the versions that do not have any children. So, that a signature of a previous version of the parent node is stored in at least one child node. Each child only aggregates the signature of two consecutive versions of the parent, so that there are only two cases of the signatures: (1) the signature is not combined with another signature, in this case the signature is stored in its original form (2) the signature is combined with another signature to form an aggregation of two signatures.

If a signature $\sigma_{i}$ is not combined, we should find the other signature of the consecutive version in other children. Because the other signature should be stored in at least one other child, if the other signature is stored as the first case (no combination with other signature), we can combine with $\sigma_{i}$ to get a combination of two consecutive signatures. If the other signature has been combined, it should have been combined with $\sigma_{i}$ (the same signatures stored in the other child), so in all cases we can combine elements in all singedge in the children to form signnode at the parent.

\section{$7 \quad$ Storage Requirements}

The storage for signnode is always constant for any number of updates to the node, which is two signatures for each node (one signature for the latest version, and a signature for aggregate of signatures of all previous versions). As of the storage for signedge, in the best case, a child aggregates all the signatures of all versions of the parent, so it only needs to store one signature for each parent. The worst case is if the child does not uses any two consecutive versions of the parent, so we cannot reduce the number of signatures in TreeAggr. In this case, the number of signatures is $n / 2$ where $n$ is the number of updates to parent.

\section{References}

1. Aldeco-Pérez, R., Moreau, L.: Securing Provenance-Based Audits. In: McGuinness, D.L., Michaelis, J.R., Moreau, L. (eds.) IPAW 2010. LNCS, vol. 6378, pp. 148-164. Springer, Heidelberg (2010)

2. Álvarez, S., Vázquez-Salceda, J., Kifor, T., Varga, L.Z., Willmott, S.: Applying Provenance in Distributed Organ Transplant Management. In: Moreau, L., Foster, I. (eds.) IPAW 2006. LNCS, vol. 4145, pp. 28-36. Springer, Heidelberg (2006)

3. Bellare, M., Namprempre, C., Neven, G.: Unrestricted Aggregate Signatures. In: Arge, L., Cachin, C., Jurdziński, T., Tarlecki, A. (eds.) ICALP 2007. LNCS, vol. 4596, pp. 411-422. Springer, Heidelberg (2007)

4. Boneh, D., Gentry, C., Lynn, B., Shacham, H.: Aggregate and Verifiably Encrypted Signatures from Bilinear Maps. In: Biham, E. (ed.) EUROCRYPT 2003. LNCS, vol. 2656, pp. 416-432. Springer, Heidelberg (2003) 
5. Braun, U., Shinnar, A., Seltzer, M.: Securing provenance. In: The 3rd USENIX Workshop on Hot Topics in Security (HOTSEC 2008), USENIX HotSec, pp. 1-5. USENIX Association, Berkeley (2008)

6. Deora, V., Contes, A., Rana, O.F., Rajbhandari, S., Wootten, I., Kifor, T., Varga, L.Z.: Navigating provenance information for distributed healthcare management. In: Web Intelligence, pp. 859-865 (2006)

7. Groth, P.T.: The Origin of Data: Enabling the Determination of Provenance in Multi-institutional Scientific Systems through the Documentation of Processes. $\mathrm{PhD}$ thesis, University of Southampton (2007)

8. Groth, P.T., Moreau, L.: Recording process documentation for provenance. IEEE Trans. Parallel Distrib. Syst. 20(9), 1246-1259 (2009)

9. Haber, S., Stornetta, W.S.: How to time-stamp a digital document. J. Cryptology 3(2), 99-111 (1991)

10. Hasan, R., Sion, R., Winslett, M.: Introducing secure provenance: problems and challenges. In: ACM Workshop on Storage Security and Survivability (StorageSS 2007), pp. 13-18 (2007)

11. Hasan, R., Sion, R., Winslett, M.: The case of the fake picasso: Preventing history forgery with secure provenance. In: 7th Conference on File and Storage Technologies (FAST 2009), pp. 1-14 (2009)

12. Hasan, R., Sion, R., Winslett, M.: Preventing history forgery with secure provenance. ACM Transactions on Storage 5(4), 12:1-12:43 (2009)

13. Kifor, T., Varga, L.Z., Vázquez-Salceda, J., Álvarez-Napagao, S., Willmott, S., Miles, S., Moreau, L.: Provenance in agent-mediated healthcare systems. IEEE Intelligent Systems 21(6), 38-46 (2006)

14. Miles, S., Groth, P.T., Munroe, S., Jiang, S., Assandri, T., Moreau, L.: Extracting causal graphs from an open provenance data model. Concurrency and Computation: Practice and Experience 20(5), 577-586 (2008)

15. Moreau, L., Clifford, B., Freire, J., Futrelle, J., Gil, Y., Groth, P.T., Kwasnikowska, N., Miles, S., Missier, P., Myers, J., Plale, B., Simmhan, Y., Stephan, E.G., Van den Bussche, J.: The open provenance model core specification (v1.1). Future Generation Comp. Syst. 27(6), 743-756 (2011)

16. Moreau, L., Freire, J., Futrelle, J., McGrath, R.E., Myers, J., Paulson, P.: The Open Provenance Model: An Overview. In: Freire, J., Koop, D., Moreau, L. (eds.) IPAW 2008. LNCS, vol. 5272, pp. 323-326. Springer, Heidelberg (2008)

17. Muniswamy-Reddy, K.-K.: Foundations for Provenance-Aware Systems. PhD thesis, Harvard University (2010)

18. Simmhan, Y., Plale, B., Gannon, D.: A survey of data provenance in e-science. In: SIGMOD Record, pp. 31-36 (2005)

19. Syalim, A., Nishide, T., Sakurai, K.: Preserving Integrity and Confidentiality of a Directed Acyclic Graph Model of Provenance. In: Foresti, S., Jajodia, S. (eds.) Data and Applications Security and Privacy XXIV. LNCS, vol. 6166, pp. 311-318. Springer, Heidelberg (2010)

20. Syalim, A., Nishide, T., Sakurai, K.: Securing provenance of distributed processes in an untrusted environment. IEICE Transactions 95-D(7), 1894-1907 (2012)

21. Tan, V., Groth, P.T., Miles, S., Jiang, S., Munroe, S.J., Tsasakou, S., Moreau, L.: Security Issues in a SOA-Based Provenance System. In: Moreau, L., Foster, I. (eds.) IPAW 2006. LNCS, vol. 4145, pp. 203-211. Springer, Heidelberg (2006) 